\title{
Bonne rentrée à tous !
}

\author{
S. Czernichow $\cdot$ J. Dargent \\ (C) Springer-Verlag France 2011
}

Une moisson d'articles sera à attendre du récent congrès de la Soffco à Lille, qui a été un grand succès de participation!

En attendant, vous trouverez un résumé du meeting lyonnais de mai sur les techniques non invasives de l'obésité et les nouvelles technologies, qui suscitent des interrogations légitimes, mais aussi une forme d'enthousiasme qu'il faut bien prendre en compte. Ce numéro sera aussi l'occasion de faire le point sur le syndrome de Prader-Willi grâce à une synthèse très détaillée $\mathrm{du} \operatorname{Pr}$. M. Tauber, mais aussi de mieux comprendre l'implication du système endocannabinoïde dans la physiopathologie de l'obésité.

Les articles chirurgicaux concerneront aussi la plicature gastrique verticale annoncée spectaculairement comme un sleeve-killer ! Un cas d'occlusion du grêle par migration d'un ballon intragastrique, un traitement faussement présenté comme anodin ; les possibilités de traitement chirurgical par by-pass biliopancréatique dans le syndrome de Prader-Willi et une étude exemplaire sur les facteurs d'adhésion thérapeutique aux suppléments vitaminiques après chirurgie bariatrique. Enfin, nous avons aussi décidé de publier les premiers résultats d'une enquête sur internet qui décrypte les pratiques de suivi du régime Dukan, chez les utilisateurs d'internet. Cette étude, dont la méthodologie peut évidemment être discutée, aura pour mérite de mettre le sujet au centre du débat et montre les besoins en matière d'évaluation scientifique des différents régimes proposés actuellement....

Bonne lecture ! 